\title{
Micromachining process - current situation and challenges
}

\author{
Meet Rajeshkumar Lalakiya ${ }^{1, a}$ \\ ${ }^{1}$ Christ University Faculty of Engineering in Bengaluru, INDIA
}

\begin{abstract}
The rapid progress in the scientific innovations and the hunt for the renewable energy increases the urge for producing the bio electronic products, solar cells, bio batteries, nano robots, MEMS, blood less surgical tools which can be possible with the aid of the micromachining. This article helps us to understand the evolution and the challenges faced by the micromachining process. Micro machining is an enabling technology that facilitates component miniaturization and improved performance characteristics. Growing demand for less weight, high accuracy, high precision, meagre lead time, reduced batch size, less human interference are the key drivers for the micromachining than the conventional machining process.
\end{abstract}

\section{Introduction}

Miniaturization is a significant trend of a widespread technology, utilized in every field. Micromachining of materials with micron resolution at high $\mathrm{s}$ nonconventional production called EDM. EDM is uses electro-thermal energy to remove the materials without friction but with the utility of electric power. It is identified as an optimized technology in the 1980's where the accuracy is so high when compared with the conventional machining process where the tool vibration, dimension, speeds of machine tool and various uncertain parameters drives the accuracy.

EDM in the 1980's are replaced by the Micro EDM's, which has its control over the plasma channel radius. The advent of micro EDM's helps the users to reach the micro hole of diameter $50 \mu \mathrm{m}$ to $100 \mu \mathrm{m}$. EDM provides seed to the invention of Laser micromachining, LIP-MM where the operator has control over multiple parameters of the work piece. The Efficiency of work piece is highly increased.

In the medical field, diagnosis and surgery with bloodless and painless is possible through the size reduction of surgical tools. The value addition and the comfort level of the users are greatly increased with reduced size and weight. With the future projection towards miniaturization, micromachining becomes more inevitable in fabricating micro parts. Micro parts may have overall size of few millimeters but it has many features that falls in micro range from $1 \mu \mathrm{m}$ to $500 \mu \mathrm{m}$. The design and construction of micro tools have increased the aspiration of the human kind more towards the automation with less human interference. It steeply cultivates the interaction between the micro toolcomputer interfaces to take the decision on its own without the human participation.

\section{EDM process and classifications}

\subsection{EDM process}

\footnotetext{
${ }^{a}$ Corresponding author: lalakiyameet@gmail.com
}

Micro EDM [1] is an advanced version of the macro EDM which can produce the micro parts of $50 \mu \mathrm{m}-100$ $\mu \mathrm{m}$. A pulse generated between the tiny gap between the electrode and the work piece through the dielectric will peel the required material from the work piece. Major difference between the micro EDM and the Macro EDM is the plasma radius. The plasma radius can be increased by the time of exposure. Micro EDM will be takes place in the lesser time intervals and vice versa.

When the two electrodes are separated by any distance, and when it is excited the insulating dielectric medium breaks down into conductive dielectric medium (ionization) to conduct heat from the electrode to the work piece. The insulating medium is used to avoid the electrolysis (breaking) of the electrode. The electrode shape is reflected on the surface of the work piece, which is separated by the distance. Discharge occurs at the frequency of 103 to $106 \mathrm{HZ}$.

After every pulse, piece of material will be flushed both from the electrode and the work piece material. It is mainly used in the die making industries. The peeled off materials are condensed into small particles called spherical debris particles.

To get the stable plasma, every new spot of machining is to be far distanced from the previous location to avoid the impact due to the contaminated spherical debris, which will slow down the dielectric breakdown.

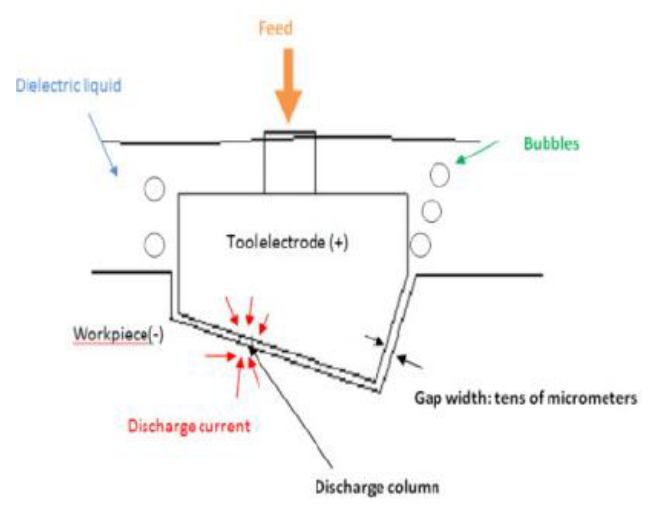

Figure 1. Concept of EDM. 


\subsection{EDM classifications}

\subsubsection{Based on the process}

Sinking EDM [1]. The work piece obtained by this process will follow any of the three forming methods, either formed by the projection of a shape tool electrode or by the 3 dimensional movement of the electrode, or the combination of the both. The Numerical controllers used for the uniform movement in the different axis and the gap conditions and so on.

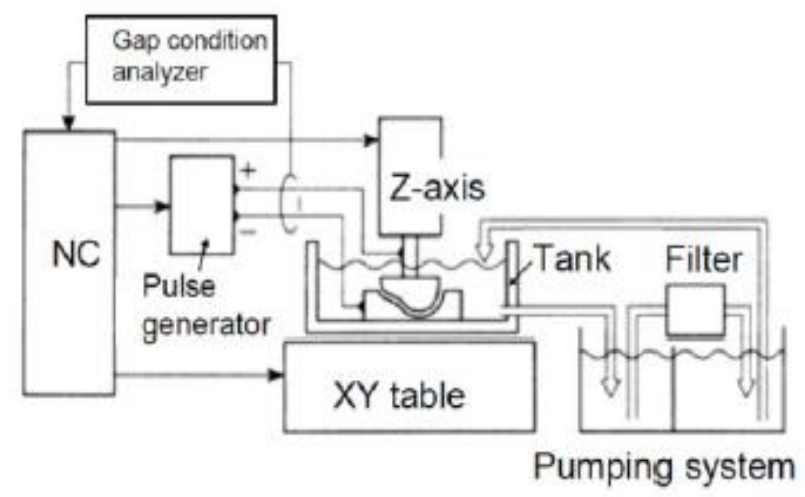

Figure 2. Sinking Electrical Discharge Machining.

Wire EDM. In wire EDM [1], the wire electrode is brass wire or coated steel wires, but in thin diameter machining, tungsten or molybdenum wires are used. A discharge with high peak value with low short duration is used. Water with reduced value of specific dielectric liquid using de-ionising resins is used to avoid electrolysis.

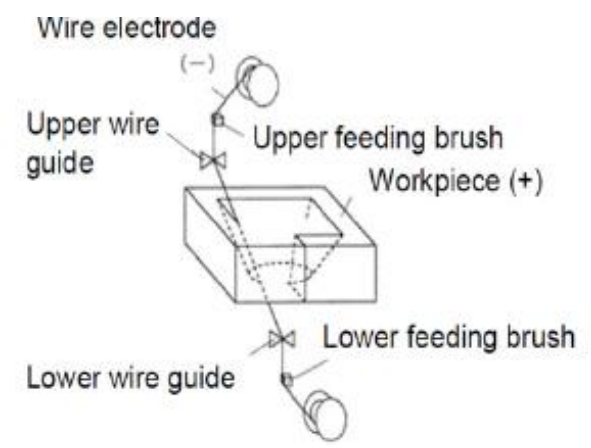

Figure 3. Wire Electrical Discharge Machining.

\subsubsection{Based on power supply}

Rotary impulse generator. In rotary impulse generator, the voltage output will be in the form of sinusoidal form using the DC motor.

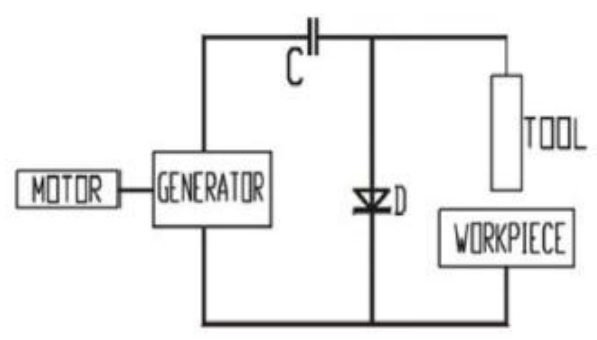

Figure 4. Rotary Impulse Generator.

Relaxation generator. In relaxation generator, the charging and the discharging cycle of the capacitor continue and it emits the saw tooth wave. The spark emited by the tool will give the charge to the capacitor whereas the ionization of the dielectric molecules will transfer the heat to the workpiece through the discharging of the capacitor.

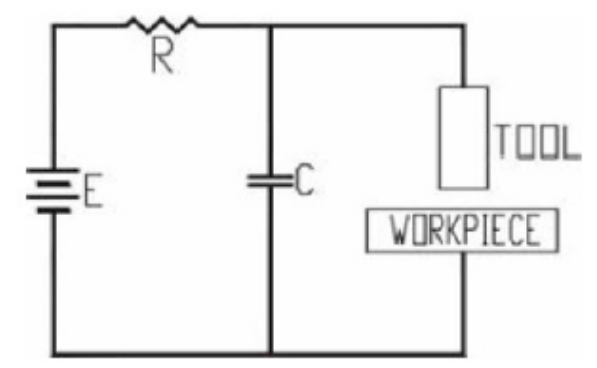

Figure 5. Relaxation Generator

\section{Laser micromachining process}

Laser micromachining [2] Process are soaring in the recent days and its potential merits add a significant impact in its application areas. Its high beam quality, portable installation size, high laser efficiency, affordable price, easy to incorporate in the system. It covers the wide utility of its usage in different fields.

Its high degree of flexibility, scope of complete automation, and its compatible installation made this process as a highly preferred one in the application industries. It is broadly classified into micro and macro machining. Micro machining is carries normal pulsed beams but operates under its operative boundary of less than 1 kilowatt. Micro machining is widely used in automobile areas as acceleration limit sensors, in medical industries for blood less surgery and it is also used in the solar cell manufacturing.

Laser micromachining is broadly classified based on the materials used for machining.

(i) Direct Laser writing

(ii) Mask Projection technique

Direct laser writing is used in the low sensitive materials. To make a concentrated beam, solid state lasers are combined with the galvanometer to reduce the beam diameter and to guide it in the right way.

Mask projection technique is used in the high sensitive materials for transferring information present in the mask to the work piece. Shorter wavelength will be 
observed by the UV absorbing pigments like titanium oxide.

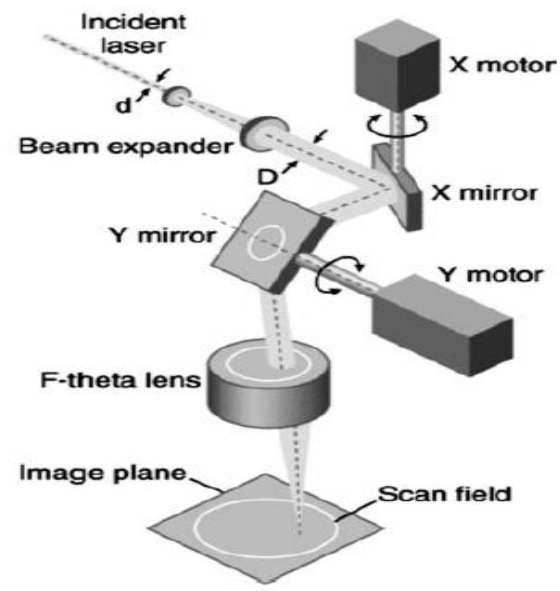

Figure 6. Direct laser writing.

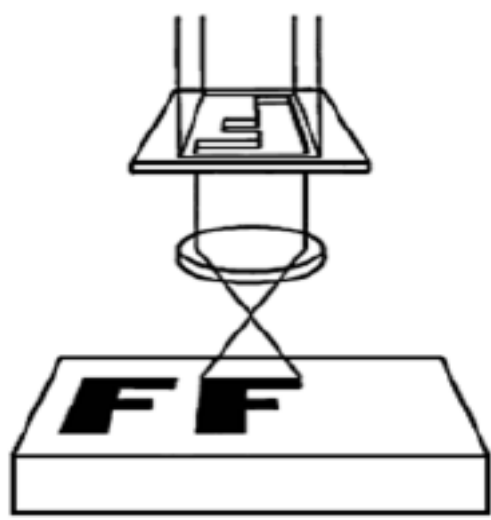

Figure 7. Mask projection technique.

\section{LIP-MM process}

LIP-MM process [3] operates on the optical absorption principle. Ultra short pulse laser beam have pulse duration from few ps (pico) to few fs (Femto) with a pulse frequent of $500 \mathrm{KHz}$ and a very low average power of $10 \mathrm{~W}$ with a very high peak power. Laser beam is tightly focused by the focused lens towards the surface of the work piece through the dielectric medium. Due to high pulse power and voltage of the laser beam, the dielectric medium got ionized and it leads to the conduction of heating from the laser beam to the surface. The plasma generated by the laser beam is transferred by the valence electrons of the dielectric medium toward the surface. This plasma leads to the liquid-gas phase change in the workpiece surface and causes cavitation in the focused surface.

\section{Challenges in micromachining}

\subsection{Challenges in EDM process}

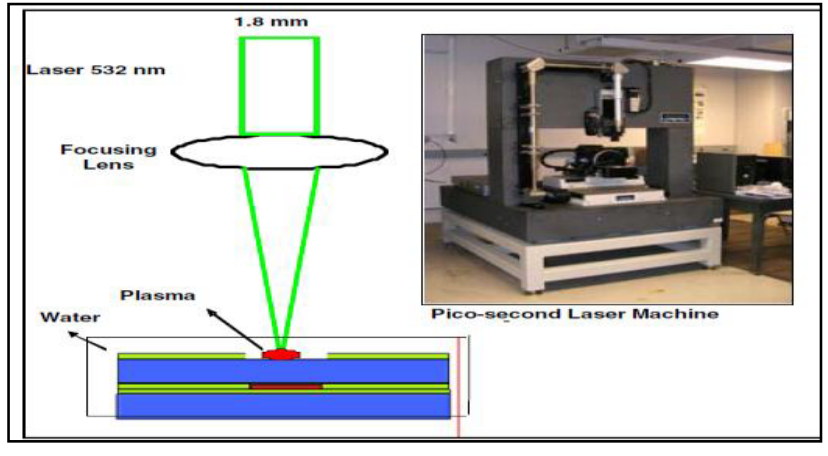

Figure 8. LIP-MM Process.

Conventional micro EDM [1] is the pioneer in the material removal process. It is generated by the plasma when high electric field is generated into the dielectric medium closer to the threshold frequency and in turn ionization will send the (electric) shock waves to the surface of the work piece that leads to the localized heating and ablation.

The challenges of micromachining are studied in the spectrum of process physics [4]. This article helps to understand the size of the work material to the tool dimension used, work piece behavior if the material removal is either isotropic or anisotropic. If the work piece is homogenous and it is isotropic, the size effect is the key factor to decide the efficiency of the toolpiece. In the anisotropic material removal, the cutting plane made to pass through the grain boundaries. Size effect is defined as the ratio of the depth of cut to the tool edge radius. The tool radius is the key aspect in of chip thickness. The low chip thickness can be attained by reduced tool radius. Minimum chip thickness can be achieved by the tool of copper and aluminum can provide $5 \%$ of its tool radius whereas the aluminum alloy tool will produce 20 to $40 \%$ of its tool radius respectively.

The limitations of micro EDM [5] are

(i) Limited to operate only in conductive materials whereas low conductive material and nonconductive material are highly inert to the EDM micro machining.

(ii) Nonproductive time and the cost to operate are relatively higher than other process.

(iii) Frequent electrode replacement is needed because of electrode wear.

(iv) Removal of debris from the least electrode gap is 0.5 $\mathrm{nm}$

(v) Meagre material removal rate $\left(0.6\right.$ to $\left.6 \mathrm{~mm}^{3} / \mathrm{hr}\right)$

\subsection{Challenges in laser micromachining process}

In the recent years, the laser micromachining process [6] is highly employed for precise monitoring and quality assurance. Nano and Picosecond lasers offer high precision for all Machin- able materials but there limitations are environment related factor, tight tolerance component, short cycle times, high uncertainty, low lateral accuracy, high material dependency. It reduces the probability of desired results. To obtain expected 
outcomes laser triangulation and capacitive sensors may be applied for process monitoring and control.

\section{Discussions and conclusion}

The challenges put forward in the micromachining are the driving factor for the research investigations of this field. The cost of operation and the maintenance is greatly reduced by the advent of the Laser optical absorption principle in the micromachining process. The Optical absorption process reduced the usage of the electrodes and the avoidance of the sedimentation of spherical debris in the work piece surface. Laser induced Plasma micro machining process be a motor for the $3 \mathrm{D}$ printing process.

In the micro EDM, the sources of the errors are identified and the trouble-shooting process is done. In the article [7], it explains the strategy of reducing the error. When the tolerances are assigned for micro EDM, the article recommends to include the parameters such as the type of electrode grinding, type of positioning and the duration of operation et al to avoid the piling of error. It also suggests that overall efficiency depends on the relationship between the process parameters and their optimization during experimental process. To achieve the high precise values, the paper suggests using the reliable algorithms, which can capture the recently utilized optimal positions, and it can be repeated in the future process.

\section{References}

1. S. Mahendran, R. Devarajan, T. Nagarajan, and A. Majdi, 'A Review of Micro-EDM', Proceedings of the international Multiconference of Engineers and Computer Scientists, ISBN: 978-988-18210-4-1, ISSN: 2078-0958 (Print); ISSN: 2078-0966(online), (2010)

2. UdoKlotzbach, A. F. Lasagni, M. Panzner and V. Franke, 'Laser micromachining', Springer-Verlag Berlin Heidelberg, ASM 10, (DOI: 10.1007/978-3642-17782-8_2), (2011)

3. K. Pallav and K. F. Ehmann, 'Feasibility of Laser Induced Plasma Micro-machining (LIP-MM)'- IPAS 2010, International Federation for Information Processing, IFIP AICT 315, pp. 73-80, (2010)

4. D. Dornfeld, S. Min, Y. Takeuchi, Recent Advances in Mechanical Micromachining by Annals of the CIRP 55, (2006) Web link: $\frac{\text { http://escholarship.org/uc/item/8rf718jm }}{\text { K. Pallav, K. F. Ehmann, Feasibility of Laser }}$ induced Plasma Micromachining, IFIP-International Federation for Information Processing, pp. 73-80, (2010)

6. R. Schmitt, G. Mallmann, Process Monitoring in Laser micromachining, Photonik international, pp 57-59, 3 (2013)

7. D. T. Pham, S. S. Dimov, S. Bigot, A. Ivanov, and K. Popov, Micro-EDM - Recent Developments and Research Issues, JMPT 149, pp. 50-57, (2004) 